\title{
Características del alumno y aprendizaje cooperativo: aplicación a la enseñanza de Economía de la Empresa
}

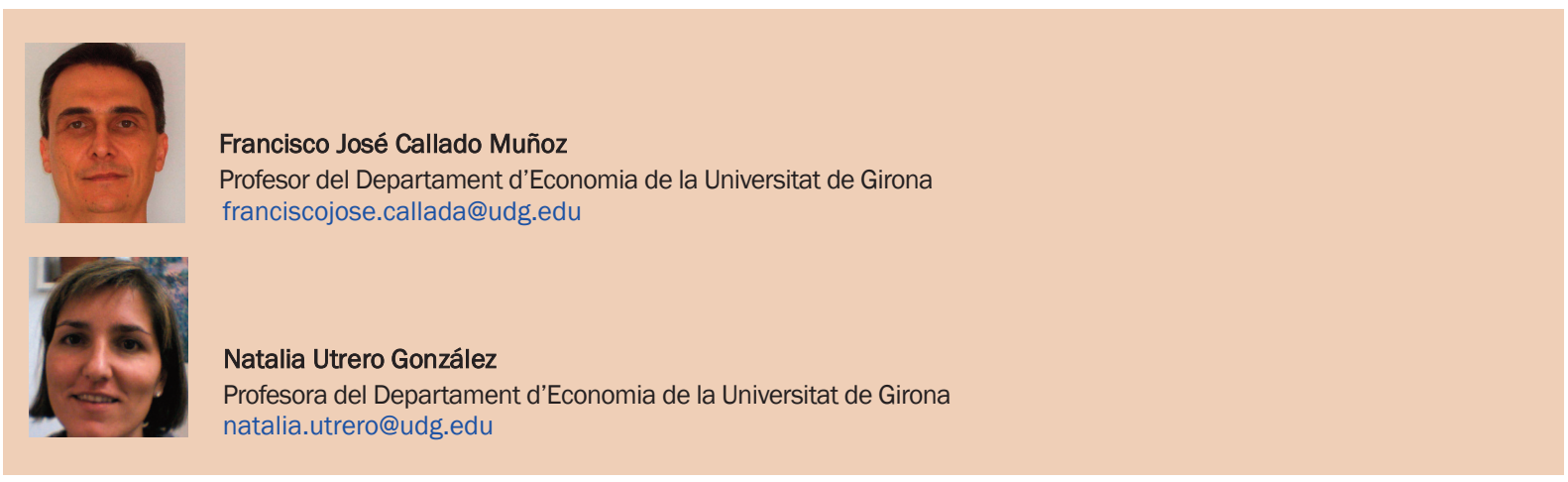

|Fecha presentación: 07/04/2011 | Aceptación: 02/02/2012 |Publicación: 20/06/2012

\begin{abstract}
Resumen
Este trabajo se centra en el aprendizaje cooperativo. Se analizan algunas características que pueden tener influencia en el éxito y buen funcionamiento de esta herramienta como instrumento didáctico dentro del nuevo contexto universitario. Se realizan experiencias de trabajo cooperativo en dos grupos de alumnos, con resultados muy distintos. Se analizarán distintas variables que pueden ayudar a explicar el porqué de este distinto comportamiento. Los resultados pretenden contribuir al conocimiento de los elementos que influyen en el éxito de este tipo de procedimientos, así como a proporcionar algunas claves que ayuden en la organización y configuración de los grupos de trabajo.
\end{abstract}

Palabras clave: aprendizaje cooperativo, organización, resultados

\section{Resum}

Aquest treball es centra en l'aprenentatge cooperatiu. S'analitzen algunes característiques que poden tenir influència en l'èxit i bon funcionament d'aquesta eina com a instrument didàctic dins del nou context universitari. Es realitzen experiències de treball cooperatiu en dos grups d'alumnes, amb resultats molt diferents. S'analitzaran diferents variables que poden ajudar a explicar el perquè d'aquest diferent comportament. Els resultats pretenen contribuir al coneixement dels elements que poden influir en l'èxit d'aquest tipus de procediments així com de proporcionar algunes claus que ajudin en l'organització i configuració dels grups de treball.

Paraules clau: aprendizaje cooperativo, organización, resultados.

\section{Abstract}

This work focuses on cooperative learning. It analyzes some of the features that can influence the success and smooth functioning of this tool as a teaching device within the new university paradigm. Some cooperative work experiences have been realized in two groups of students with very different results. We analyze a given set of variables that may help explain this different behavior. The results try to contribute to the knowledge of factors which may influence the success of such procedures as well as provide some clues to help in the organization and distribution of students in different groups.

Keywords: cooperative learning, organization, results 


\section{Introducción}

Algunos de los cambios a los que se enfrenta la enseñanza superior en los próximos años tienen que ver con la creación del Espacio Europeo de Educación Superior (Gainza 2003). Se trata por tanto de afrontar los desafíos generales dentro de un marco europeo y con unos objetivos comunes a alcanzar.

Uno de los elementos que aparecen cuando se habla de la adaptación al Espacio Europeo de Educación Superior (EEES) es la de propiciar un cambio en el concepto de aprendizaje de transmisor (profesor) a receptor (alumno) como dos entidades diferenciadas. Se debe evolucionar hacia un concepto de guía (el profesor conducirá) para la construcción activa de conocimiento por parte del alumno. Se trata, por tanto, de la construcción progresiva y conjunta de sistemas de significación compartidos entre ambos y de un traspaso gradual del control y la responsabilidad del aprendizaje del profesor al alumno. Uno de los procedimientos que propician este cambio son las experiencias de innovación educativa basadas en el aprendizaje colaborativo (Johnson et al. 1991; Jonson y Johnson 1999).

En muchas ocasiones las experiencias de innovación docente son difíciles de realizar en el contexto universitario con presencia de distintos grupos de alumnos muy numerosos y en ocasiones a cargo de profesores diferentes. De esta manera la coordinación es compleja y suele estar centrada en objetivos de aprendizaje (Bohigas 2009).

La experiencia que presentamos en este trabajo se realizó en una situación como la que acabamos de exponer, en la que la actividad del profesor está centrada en una programación y coordinación de corte tradicional. La actividad se plantea con el objetivo de que los alumnos participen más activamente en el proceso de aprendizaje. En este contexto, la motivación de este estudio surge a partir de una serie de experiencias de aprendizaje cooperativo realizadas dentro de la asignatura "Introducción a la Economía Política" de primer curso de la diplomatura de Empresariales de la Facultad de Económicas de la Universitat de Girona durante el curso académico 2004/2005. Esta asignatura se divide en dos cuatrimestres: el primero de introducción a la economía y el segundo de introducción a la economía de la empresa que es donde se realiza este ejercicio. En particular, se propone realizar actividades en clase (con gran número de alumnos) para fomentar el trabajo cooperativo y mejorar el proceso de aprendizaje. Los resultados de estas actividades tuvieron un efecto positivo en el aprendizaje del alumnado, sobre todo en una de las clases donde se realizó. Partiendo de esta actividad y su diferente resultado, se plantea la siguiente pregunta: ¿de qué depende el grado de éxito de este tipo de experiencias? La hipótesis de trabajo es que puede haber características particulares del alumnado que faciliten el buen funcionamiento y resultado de estas actividades de innovación. El objetivo de este trabajo sería por tanto el de realizar un análisis de un determinado grupo de variables que pueden ayudar a explicar el porqué de este distinto comportamiento. Los resultados de este análisis pretenden contribuir al conocimiento de los elementos que pueden influir en el éxito de este tipo de procedimientos, así como de proporcionar algunas claves que ayuden en la organización y distribución del alumnado en diferentes equipos de trabajo.

A continuación se explica como se ha realizado esta experiencia docente y en qué ha consistido.
2. Descripción de la experiencia y realización del estudio A modo de ejemplo se presenta una de las actividades que se desarrollaron a lo largo del curso

Título de la Actividad: ¿Coordinación económica por la empresa o por el mercado?

El tema a tratar se refiere a la existencia de dos modos de coordinación de una actividad económica: mercado y/o empresa. Esta elección depende, entre otras muchas variables, de la presencia o no de una serie de factores tales como la existencia de economías de escala, el número de oferentes en el mercado, la frecuencia de las transacciones etc. Todos estos conceptos se han definido en clase previamente a la realización de la actividad. Además los alumnos tienen a su disposición material docente de apoyo.

Ante la presencia de cada uno de estos factores la pregunta a contestar por los alumnos es: ¿debemos organizar la actividad económica a través de un sistema de mercado o conviene organizarla dentro de la empresa? Se debe completar por parte de cada equipo de trabajo la tabla que aparece a continuación, indicando en cada factor cuál de los dos sistemas es el más adecuado.

\begin{tabular}{|c|l|l|}
\hline Factores & Mercado & Empresa \\
\hline Economías de escala y alcance & & \\
\hline Muchos oferentes & & \\
\hline Pocos oferentes & & \\
\hline Oportunismo & & \\
\hline Activos Específicos & & \\
\hline $\begin{array}{c}\text { Conocimientos Específicos } \\
\text { de la Empresa }\end{array}$ & & \\
\hline Incertidumbre & & \\
\hline Frecuencia de las Transacciones & & \\
\hline
\end{tabular}

La organización de este tipo de actividades en equipo recoge los parámetros que definen la metodología cooperativa (Johnson y Johnson 1991): Criterio de éxito: cualquier miembro del equipo será capaz de explicar las características que se pueden presentar en el entorno de negocios y como se debe decidir entre mercado y empresa ante cada situación. Interdependencia positiva: cada miembro del equipo tiene una parte del material y un rol asignado para que la tarea funcione bien. Exigibilidad personal: Un miembro del equipo elegido al azar responderá a las preguntas del profesor sobre el material. Habilidades sociales en juego: capacidad para comprender conceptos y analizar situaciones que ayuden en la toma de decisiones. Cuestionar y pedir explicaciones sobre las intervenciones de otros Reflexión sobre el trabajo en equipo: cada miembro del equipo debe mencionar una cosa que ha ido bien y que ha ido mal en el trabajo del equipo (ver ficha en el anexo).

En las primeras sesiones los estudiantes están reticentes hacia este tipo de metodología; sin embargo, conforme el curso avanza, se sienten más cómodos y participativos. La mayoría destaca, al final, lo útil que las actividades son para clarificar conceptos y poder aplicarlos a casos y ejemplos más cercanos a la realidad.

Por otro lado, la realización continuada de actividades de aprendizaje cooperativo con el mismo equipo ayuda a conseguir mayor interdependencia entre los miembros de 
los equipos, es decir, aumenta el grado en el que los miembros se necesitan unos a otros para poder completar con éxito las tareas asignadas (Barrick et al. 2007). De hecho, como se ha destacado en otros trabajos, la interdependencia marca la diferencia entre un equipo y un grupo, fundamental para la correcta implementación de la metodología de trabajo cooperativo (Iborra y Dasí 2009).

La experiencia se lleva a cabo en dos de los grupos distintos de alumnos (grupos C y D). Ambos grupos tienen horario de tarde y se componen de 59 y 66 alumnos respectivamente. Son alumnos de distinta procedencia académica, que en su gran mayoría trabajan y compaginan su horario laboral con la formación universitaria. En el grupo $\mathrm{C}$ las actividades de aprendizaje cooperativo llevadas a cabo tuvieron resultados mucho mejores que en el grupo D. Además de los resultados, la composición de los grupos es muy diferente.

A partir de esta evidencia nos preguntamos si se puede caracterizar de algún modo al alumno que se integra de una manera más adecuada en este tipo de procedimientos de aprendizaje y que es capaz de realizar un mayor aprovechamiento de estas actividades. Para ello, se ha recopilado la información que sobre los alumnos posee el área de gestión académica de la facultad. En estos datos se dispone tanto de los resultados académicos como de diversas características personales del alumnado.

\section{Datos y Análisis}

Las condiciones que se deben cumplir para realizar un buen trabajo cooperativo son entre otras, una serie de aptitudes individuales, como habilidades de intercambio personal y en grupo (Johnson et al. 1999a; 1999b). Estas características podrían estar relacionadas con algunas de las características de partida del alumno. Por tanto, el primer paso es analizar las distintas características personales del alumnado. Las características que se analizan son: sexo, edad, calificación y tipo de acceso a la universidad, calificación media del expediente universitario, número de convocatorias gastadas en la asignatura objeto de estudio. La metodología de análisis de esta información se ha dividido en dos fases:

Fase 1: Se calculan estadísticos descriptivos de las distintas variables y se ha estudiado si existen diferencias sig-

\begin{tabular}{|c|c|c|}
\hline & \multicolumn{2}{|c|}{ Medias y Proporciones } \\
\hline Variable & $\begin{array}{c}\text { Grupo C } \\
\text { (59 alumnos) }\end{array}$ & $\begin{array}{c}\text { Grupo D } \\
\text { (66 alumnos) }\end{array}$ \\
\hline \% hombres & 0.47 & 0.38 \\
\hline \%mujeres & 0.53 & 0.62 \\
\hline Edad & 23.32 & 22.71 \\
& $(0.5635)$ & $(.5587)$ \\
\hline Calificación acceso* & 1.63 & 1.76 \\
& $(0.7856)$ & $(1.0386)$ \\
\hline \multirow{2}{*}{ Media expediente* } & 1.26 & 1.22 \\
& $(0.4691)$ & $(0.5750)$ \\
\hline \multirow{2}{*}{ Num. Convocatorias } & 1.53 & 1.33 \\
& $(-1.1041)$ & $(0.7712)$ \\
\hline Nota & $7.24^{*}$ & $6.40^{*}$ \\
& $(1.7134)$ & $(1.7470)$ \\
\hline
\end{tabular}

Tabla 1. Estadísticos descriptivos y Diferencia de medias entre los grupos. Los números entre paréntesis indican la desviación estándar. * diferencias significativas de la variable en cuestión entre los grupos $C$ y D. *Calificación de acceso y media del expediente calculada sobre 5 nificativas entre los grupos C y D del curso 2004-2005. Fase 2: Se ha tratado de comprobar si los resultados obtenidos en las actividades de trabajo cooperativo pueden ser explicados por dichas características de los individuos que forman parte de cada uno de los grupos. En concreto se ha regresado la calificación del aprendizaje cooperativo con las distintas variables disponibles. De este modo se trata de establecer la relevancia de cada una de ellas en el desempeño de actividades de este tipo.

En el grupo $\mathrm{C}$ las actividades de aprendizaje cooperativo llevadas a cabo tuvieron resultados mucho mejores que en el grupo D. Por tanto, analizaremos y realizaremos la comparación entre ambos grupos.

Fase 1.

En la tabla 1 se presenta la estadística descriptiva de las variables analizadas. Al final de la tabla aparece también la nota media de las actividades de aprendizaje cooperativo.

El sexo se ha constatado como una característica que puede tener influencia en los resultados educativos (MEC 2007) y por tanto en los resultados del grupo en su conjunto. La proporción de mujeres en un determinado grupo podría por tanto mejorar su rendimiento. Se puede observar que el grupo C presenta un menor porcentaje de mujeres frente al D. Según este indicador estaríamos en la situación contraria: un menor porcentaje de mujeres podría estar detrás del mejor rendimiento en actividades cooperativas. Sin embargo, esta diferencia de proporciones no es significativa desde el punto de vista estadístico. La edad puede también condicionar la participación y la interacción entre alumnos en ejercicios cooperativos. Una mayor edad supone un mayor grado de madurez que puede favorecer la discusión y puesta en común de argumentos. En este caso el grupo C presenta una media de edad mayor que el grupo D. La diferencia de proporciones estaría de acuerdo con la afirmación anterior pero de nuevo la diferencia no resulta significativa.

Otra variable interesante sería el modo de acceso a los estudios (ver Gráfico 1). En este caso, se observa que la proporción de estudiantes que provienen de otros estudios -a los que accedieron desde el Curso de Orientación Universitaria (COU)- es mayor en el grupo C frente al D. Lo contrario ocurre en el caso de los alumnos procedentes de Formación Profesional (FP). El resto de procedencias tienen proporciones similares. Sin embargo, esta diferencia en el modo de

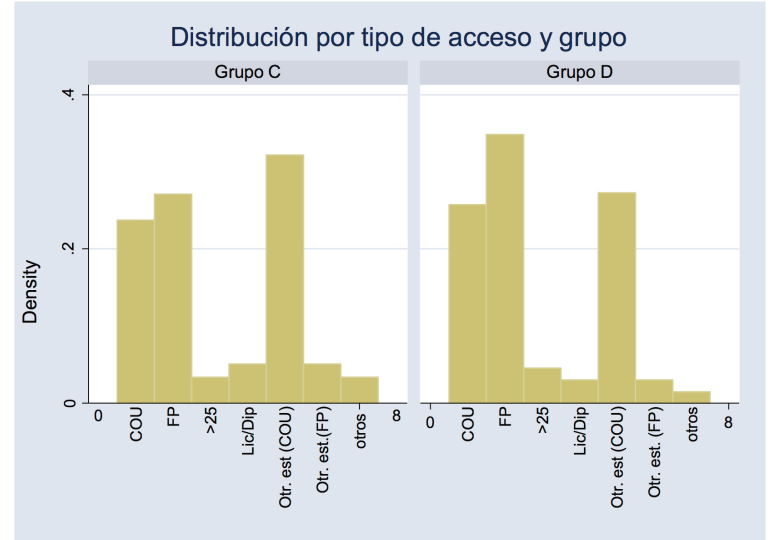

Gráfico 1. Modo de acceso a los estudios. Lectura. COU: procedentes de COU, FP: procedentes de FP, >25: mayores de 25, Lic/Dip: Licenciados o Diplomados, Otr. Est. (COU): Otros estudios con procedencia de COU, Otr. Est. (FP): Otros estudios con procedencia de FP, otros: otros accesos. 


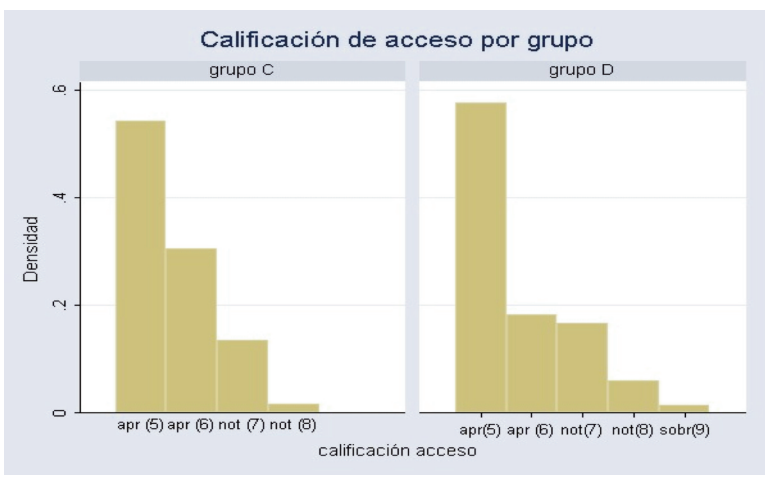

Gráfico 2. Calificación obtenida para el acceso. Lectura: apr(5): aprobado con calificación de 5 , apr(6): aprobado con calificación de 6 , not(7): notable con calificación de 7, not(8): notable con calificación de 8, sobr (9): sobresaliente con calificación de 9.

acceso no resulta tampoco significativa.

Un aspecto relacionado con el modo de acceso es la calificación obtenida para el acceso. En este caso se observa una distribución distinta entre grupos (Ver Gráfico 2). La proporción de aprobado (5) en el grupo D es mayor que en el C mientras que en el caso de aprobado con un 6 ocurre lo contrario. En la parte alta de la distribución el grupo D tiene proporciones mayores. Sin embargo, el análisis de la media de la distribución de las calificaciones de acceso indica que no hay diferencias significativas entre los dos grupos (tabla 1).

En el caso de la nota media del expediente en la diplomatura y el número de convocatorias empleadas en aprobar la asignatura ambos presentan valores mayores en el grupo C. El nivel medio del estudiante parece mayor aunque se necesitan un mayor número de convocatorias para superar la asignatura. De nuevo, estas diferencias no resultan significativas. Por último se analiza la nota de la parte de la asignatura que incluye los ejercicios de aprendizaje cooperativo. En este caso el grupo C presenta mejores resultados y ahora sí son significativos desde el punto de vista estadístico. Por tanto, el desempeño en este tipo de actividades presenta una diferencia entre los dos grupos

Fase 2.

A partir de este estudio descriptivo se plantea realizar una regresión de los resultados de las actividades de aprendizaje cooperativo con las características personales analizadas. La tabla 2 presenta las correlaciones entre ellas. En general no existen correlaciones significativas. Tan solo aparecen tres casos. Primero, correlación negativa entre la nota de las actividades cooperativas y el número de convocatorias utilizadas para superar la asignatura. Es decir, cuantas más oportunidades se necesitan para superar la asignatura menor es el rendimiento obtenido en las actividades de aprendizaje cooperativo. Con el mismo signo aparecen el número de convocatorias con la nota de acceso. Parece que el desempeño en la educación previa a la universidad condiciona el número de convocatorias necesarias. De signo positivo es la correlación en la nota de las actividades cooperativas y la calificación media del expediente. Un mejor expediente supone mejores resultados en la asignatura.

Los resultados de la regresión aparecen en la Tabla 3. Se trata de determinar qué variables tienen poder explicativo sobre el desempeño en actividades de aprendizaje cooperativo. Para ello se han codificado las características anteriormente comentadas. Así, el modo de acceso a los estudios se ha convertido en una variable con siete posibles valores (tantos como tipo de acceso), siendo mayor el valor de la variable cuando se aleja del cauce tradicional de entrada a los estudios universitarios. Las cuatro primeras variables: sexo (ser mujer), edad, modo de acceso y nota de acceso tienen coeficientes positivos, pero no son significativos. Una de las características que explica parte de la nota que incluye las tareas cooperativas es el número de convocatorias. Cuanto menor es el número de convocatorias que necesita un alumno para superar la asignatura mejor es su participación en estas tareas. Este resultado también podría estar indicando que los alumnos nuevos afrontan este tipo de actividades cooperativas con mayor interés frente a los que ya han cursado alguna vez la asignatura. Estos últimos podrían tener cierto cansancio y ser más reticentes frente este tipo de actividades. El grupo al que pertenece el alumno también es relevante. Tal como está definida la variable, el signo negativo indica que pertenecer al grupo $\mathrm{C}$ mejora el rendimiento del alumno en las actividades cooperativas. Además, esta variable estaría recogiendo todos los factores que tienen que ver con un posible tratamiento distinto en cada grupo (experiencia previa en la actividad, relación profesoralumno distinta, etc.). La nota del expediente tiene un efecto positivo. Por tanto, los mejores alumnos suelen ser también los que mejor se integran en este tipo de metodología de aprendizaje. De hecho, esta variable ha venido siendo utilizada en la agrupación de los alumnos con buenos resultados (Gil-Montoya et.al. 2009). Finalmente la constante es significativa con efecto positivo. Esto nos podría indicar que existen variables adicionales que pueden explicar en parte la nota en estas actividades.

\section{Conclusiones}

La motivación de este trabajo aparece a partir de una serie de experiencias de aprendizaje cooperativo en distintos grupos de una misma asignatura durante el curso académico 2004-2005. Tanto la composición de los grupos como los resultados de estas actividades de trabajo cooperativo son

\begin{tabular}{|c|c|c|c|c|c|c|c|c|}
\hline & Nota & Sexo & Edad & Acceso & Nota Acceso & N $^{\circ}$ Convoc. & Grupo & Nota Exped. \\
\hline Nota & 1 & & & & & & & \\
\hline Sexo & 0.037 & 1 & & & & & & \\
\hline Edad & -0.031 & -0.106 & 1 & & & & & \\
\hline Acceso & 0.221 & -0.106 & $0.244^{*}$ & 1 & & & & \\
\hline Nota Acceso & 0.193 & 0.120 & -0.051 & -0.050 & 1 & & & \\
\hline $\mathrm{N}^{0}$ Convoc. & $-0.348^{*}$ & 0.025 & 0.115 & $-0.273^{*}$ & -0.174 & 1 & & \\
\hline Grupo & -0.239 & 0.096 & -0.068 & -0.103 & 0.070 & -0.102 & 1 & \\
\hline Nota Exped. & $0.458^{*}$ & 0.087 & -0.005 & 0.166 & 0.217 & -0.087 & -0.045 & 1 \\
\hline
\end{tabular}

Tabla 2. Correlaciones. * Significativo al $1 \%$. 


\begin{tabular}{|c|c|}
\hline Variable & Coeficiente \\
\hline Sexo & $\begin{array}{c}0.4982 \\
(0.3715)\end{array}$ \\
\hline Edad & $\begin{array}{c}0.0076 \\
(0.0382)\end{array}$ \\
\hline Acceso & $\begin{array}{c}0.1496 \\
(0.1156)\end{array}$ \\
\hline Nota Acceso & $\begin{array}{c}0.1058 \\
(0.1937)\end{array}$ \\
\hline No Convoc. & $\begin{array}{c}-0.3965^{* *} \\
(0.1897)\end{array}$ \\
\hline Grupo & $\begin{array}{c}-0.9848^{* * * *} \\
(0.3593)\end{array}$ \\
\hline Nota Exped. & $\begin{array}{c}2.1134^{* * *} \\
(0.5818)\end{array}$ \\
\hline Constante & $\begin{array}{c}4.9760^{* * *} \\
(1.3695)\end{array}$ \\
\hline $\mathrm{R}^{2}=0.3664$ & $\mathrm{~N}=74$ \\
\hline
\end{tabular}

Tabla 3. Regresión. **significativo al $5 \%, * * *$ significativo al $1 \%$

muy diferentes. A partir de este resultado tan dispar, el objetivo de este trabajo es el estudio de los determinantes que pueden explicar este diferente comportamiento. Para ello se analizan una serie de características de los alumnos que podrían estar detrás de estas diferencias.

Los resultados del trabajo sugieren que, características como el sexo o la edad del alumno, que se han demostrado relevantes en estudios previos sobre los rendimientos académicos (MEC 2007), no son tan importantes en relación al aprendizaje cooperativo, al menos en la muestra analizada. Los rendimientos académicos anteriores tampoco condicionan los resultados de este método. En cambio, el rendimiento y las actitudes presentes frente a los estudios (convocatorias, expediente) si afectan al desarrollo de estas actividades. Por tanto, si se quiere mejorar la implementación de estas metodologías de aprendizaje se deberían tener en cuenta a la hora de confeccionar los equipos de trabajo, para mejorar el desarrollo y aprovechamiento de estas actividades. Asimismo, introducir conjuntamente prácticas que afecten positivamente al desempeño global del alumno. .

Otra de las variables que resultan relevantes es la que caracteriza a una clase frente a otra de manera general. Dentro de este concepto se puede encontrar el orden de realización de las actividades, la relación profesor-alumno etc. En el primer caso, la evidencia indica que la experiencia previa (por parte del profesor) con una determinada actividad mejora los resultados en realizaciones posteriores. Por tanto una buena preparación y la realización de estas actividades irán mejorando los resultados. En el caso de variables relacionales, es más difícil establecer recomendaciones, porque depende de cuestiones personales e incluye también la disposición del alumno. En todo caso es también importante que se cree un buen clima dentro de cada equipo, porque puede traducirse en un mejor desempeño en trabajos cooperativos. Un mejor clima no quiere decir pensamiento único, puesto que se ha evidenciado que la discusión mejora el aprendizaje cuando se utiliza este tipo de metodologías (Bohigas 2009). Por tanto, un buen clima debe fomentar la discusión de los equipos, la confianza para discrepar con el resto de los compañeros, así como los mecanismos para su- perar los conflictos (del Canto et al. 2009). De hecho, esta discusión y discrepancia fue una de las características cualitativas observadas que diferenciaba a los dos grupos.

Los resultados obtenidos son interesantes por dos motivos. En primer lugar, a partir de estos resultados se pueden plantear futuras investigaciones con un enfoque más global que incluya una mayor información sobre el alumno, incluso realizar el seguimiento de un determinado grupo de alumnos a su paso por la facultad, así como la utilización de otras técnicas que enriquezcan las conclusiones. Por ejemplo, las técnicas de análisis multivariante pueden dar sentido al efecto de un conjunto de variables en el desempeño en las actividades de aprendizaje cooperativo. A pesar de que de manera individual algunos factores no resultan relevantes, podrían, de manera conjunta, tener algo que decir. Además, la realización de encuestas en el alumnado antes y después de las experiencias de trabajo cooperativo puede ayudar a identificar nuevas dimensiones que tengan relevancia para el tema tratado. En segundo lugar, creemos que los resultados presentados pueden ser de utilidad a otros profesores a la hora de diseñar los equipos para mejorar el rendimiento de este tipo de metodologías.

\section{Bibliografía}

Barrick, Murray R; Bradley, Bret H. y Colbert, Amy E. (2007). The moderating role of Top Management Team interdependence: implications for real teams and working groups. Academy of Management Journal, 50 (3), 544-557.

Bohigas, Xavier (2009). La discusión entre compañeros mejora el aprendizaje de los estudiantes universitarios. Revista de Formación e Innovación Educativa Universitaria, 2 (1), 1-8.

Canto, Pablo del; Gallego, Isabel; López, José Manuel; Mora, Javier; Reyes, Angélica; Rodríguez, Eva; Kanapathipillai, Sanjeevan; Santamaría, Eduard y Valero Miguel (2009). Conflictos en el trabajo en grupo: cuatro casos habituales. Revista de Formación e Innovación Educativa Universitaria, 2 (4), 211-226.

De Miguel, Encarnación y Álvarez-Otero Rosa (2009). Experiencias de trabajo colaborativo en materias de ámbito científico con elevado contenido práctico. Revista de Formación e Innovación Educativa Universitaria, 2 (4), 207-210.

Gainza, Ángel María (2003). El profesor universitario en el siglo XXI. En Monereo, Carles y Pozo, Juan Ignacio (Eds). La universidad ante la nueva cultura educativa. Madrid: Síntesis, pp. 119-140.

Gil Montoya, María Dolores; Gil Montoya, Consolación; Herrada, Rosario Isabel; Gómez Julio; López, Antonio; Rebolloso, María del Mar; Novas, Nuria (2009). Aprender a cooperar y cooperar para aprender: tanto monta, monta tanto. En Gil Montoya, Ma Dolores; Ramírez Álvarez, Ma Isabel y Gil Montoya, Consolación (Eds). IX Jornadas de Aprendizaje Cooperativo y II Jornadas de Innovación Docente, Almería: Universidad de Almeria, pp. 235-244.

Iborra, María y Dasí, Ângels (2009). ¿Cómo aprender a trabajar en equipos integrados? El papel del aprendizaje cooperativo en la formación de directivos. @tic Revista d'Innovació Educativa, 2, pp.29-38. http://ojs.uv.es/index.php/attic/article/view/83 Johnson, David; Johnson, Roger (1999). Aprender juntos y solos. Aprendizaje cooperativo,

Competitivo e individualista. Buenos Aires: Aique. 
Johnson, David; Johnson, Roger; Jonhson Edyth (1999a). Los nuevos círculos de aprendizaje. La Cooperación en el aula y la escuela. Buenos Aires: Aique.

Johnson, David; Johnson, Roger; Jonhson Edyth (1999b). El aprendizaje cooperativo en el aula. Barcelona: Paidós.

Johnson, David; Johnson, Roger; Smith, Karl (1991). Cooperative learning: increasing collage faculty instructional productivity, vol. 20, no. 4.

MEC (2007). Panorama de la Educación, Indicadores de la OCDE, Informe Español. Madrid: Ministerio de Educación y Ciencia

http://www.educacion.gob.es/dctm/ievaluacion/intern acional/infoocde.pdf?documentId=0901e72b80110472

\section{Anexo.}

Plantilla de la actividad de aprendizaje cooperativo: ¿Coordinación económica por la empresa o por el mercado?

Tema: ¿Coordinación económica por la empresa o por el mercado?

Objetivos Formativos: los alumnos serán capaces de explicar las diferentes características que se pueden presentar en el entorno de los negocios y como influyen en la decisión de realizar una actividad a través del mercado o a través de la empresa.

Tamaño de los grupos: 4 Personas

Materiales: Definiciones de las diferentes características que se pueden presentar en el entorno de negocios. A cada miembro del grupo se le entregará las definiciones de dos características.

Tareas del grupo: Analizar y discutir las características utilizando el método del puzzle y decidir qué sistema de organización se debe utilizar ante cada una de las características.

Roles: Para la reunión del grupo (rotan en función de que esquema se está debatiendo):

Rol 1: Explica una situación y cual sería su decisión

Rol 2 y 3: Realiza preguntas, pide aclaraciones y comenta su opinión

Rol 4: Controla el tiempo

Organización temporal: Lectura y reflexión individual del material 5'; Discusión de las situaciones: 20'; Elaboración del Cuadro de Decisiones: 10’. 